\title{
Visual Symmetry Recognition by Pigeons
}

\author{
Juan D. Delius and Brigitte Nowak \\ Experimentelle Tierpsychologie, Psychologisches Institut, Ruhr-Universität, \\ D-4630 Bochum, Federal Republic of Germany
}

\begin{abstract}
Summary. Pigeons learned to discriminate a large number of bilateral symmetric and asymmetric visual patterns successively projected on the pecking-key of an operant conditioning chamber. Responses to the positive stimuli were reinforced according to a variable interval schedule. Once acquisition was complete generalization trials, involving sets of new stimuli, were instituted under extinction. The birds classified these novel test stimuli with high accuracy throughout, according to their symmetry or asymmetry. Their performance was not disturbed by sets of test stimuli whose geometrical style differed considerably from the training stimuli. Pigeons were even able to discriminate when only allowed the use of one eye. The generalization series were partly designed to test some classical symmetry recognition theories. None was found to be adequate. Subsidiary experiments suggested that most pigeons have a slight spontaneous preference for asymmetric patterns and that symmetry/asymmetry differences can aid pattern discrimination learning at an early stage. It is concluded that pigeons, much like humans, can discriminate bilaterally symmetric from nonsymmetric visual forms in a concept-like, generalizing way. The ontogenetic and phylogenetic development of this competence is considered. A novel symmetry recognition hypothesis based on spatial frequency analysis and neuronal featuredetector considerations is proposed.
\end{abstract}

\section{Introduction}

Symmetrical visual patterns without doubt have a singular perceptual status in humans. This is suggested by the widespread use of symmetry in art but is also confirmed by extensive experimental research. Bilateral symmetrical patterns especially have such salience although the reasons for this are not well-understood (Corballis and Beale 1976). Whether such symmetry has a particular perceptual standing with other animals is less certain. The issue is of wider interest because the demonstration of symmetry recognition in a non-human species would doubtlessly facilitate a neurophysiological

Offprint requests to: Juan D. Delius at the above address 
analysis of the phenomenon. Also, symmetry is an abstract property of certain visual forms. Even though there is no complete agreement about it, abstraction is considered to be the essence of perceptual concepts and these are traditionally viewed as a cognitive, exclusively human competence (but see for example Herrnstein and de Villiers 1980).

Following preliminary evidence that, in contrast to an earlier report (Morgan et al. 1976), pigeons can learn to distinguish symmetrical from asymmetrical patterns in a concept-like way (Delius and Habers 1978) we now report more detailed research into this question. Four experiments were performed. A preference test examined whether pigeons have a consistent, unconditioned spontaneous preference for either symmetric or asymmetric patterns. If they did, it would suggest that they later only learned to apply this competence. The main two experiments were an extension and elaboration of our earlier effort. During a discrimination training pigeons learned to distinguish sets of symmetric and asymmetric patterns and were then tested in generalization series as to how well they would transfer this distinction to novel sets of symmetric and asymmetric patterns. The design of some of these patterns and some of the test conditions differed markedly from those used during training. This was partly an attempt to delimit the extent of the generalization to different types of stimuli and partly an effort to test two traditional hypothesis about symmetry recognition mechanisms. One of them assumes that the pictorial redundancy of symmetrical patterns is the relevant cue, the other proposes that the bilateral symmetry of the visual system is the essential feature. A final experiment involved a discrimination acquisition comparison between a group of pigeons that had to learn to distinguish between a set of symmetric and a set of asymmetric patterns (concept) and another group of pigeons that had to distinguish among two arbitrarily divided sets of patterns (control). It was intended to reveal whether experimentally naive pigeons could capitalize on a possibly already present symmetry-recognition mechanism at an early point of the differentiation learning.

\section{Methods}

Thirty adult pigeons (Columba livia) of local homing stock were employed as subjects. They were maintained at $80 \%$ of their normal weight throughout the experiments. A single-key Skinner-box of conventional design was employed. Modular digital programming and counting equipment was used to control and record all relevant events. Stimuli were back-projected onto the response key with the aid of an automatic slide projector. The projector was equipped with photocells that sensed the presence or absence of coding perforations in especially made slide frames. Patterns were drawn in black on white paper and photographically reduced negatives were affixed to the frames. The figures apeared as white stimuli of about $10 \mathrm{~mm}$ by $10 \mathrm{~mm}$ on the dark background of the $25 \mathrm{~mm}$ diameter key.

In daily 30 min sessions the pigeons were shaped to peck the key with an autoshaping procedure. At $30 \mathrm{~s}$ intervals a $5 \mathrm{~s}$ illumination of the key was followed by $3 \mathrm{~s}$ of grain access. Then the subjects were transferred to a continuous reinforcement schedule where the key was continuously illuminated and each peck yielded food. As soon as key-pecking was regular, a variable interval schedule was instituted. Its mean interval was gradually lengthened until the animals responded steadily on a VI $60 \mathrm{~s}$ schedule. The pretraining required some 15 sessions. 
Preference Test: The pigeons were tested for spontaneous preferences for symmetric and asymmetric patterns. Within each session 20 symmetric stimuli and 20 asymmetric stimuli were presented each for $30 \mathrm{~s}$ in an alternate sequence. The stimuli were a subset of those later used in discrimination training. The first 40 stimuli shown in Fig. 1a were employed. There was no differential reinforcement: the VI 60 s reinforcement schedule continued to be in force irrespective of the stimulus presented at any given time. Responses to the two kinds of stimuli were registered on separate counters. Group A consisting of 6 pigeons was exposed to 15 testing sessions, group B consisting of 4 pigeons to 5 such sessions.

Discrimination Training: The same pigeons plus a further group C, consisting of four pigeons, were subjected to a successive free operant discrimination learning paradigm. Sessions were daily and each consisted of the presentation of 24 stimuli, half of them symmetric, the other half asymmetric (Fig. 1a, left), ordered in varying quasi-random sequences according to Gellermann (1933). The subjects were divided into two approximately matched groups according to their performance in the preference tests. One group was assigned the symmetric, the other group the asymmetric stimuli as positive, the converse category in each case serving as negative stimuli. The positive stimuli were each presented for $30 \mathrm{~s}$, and responses to them were reinforced according to a VI $60 \mathrm{~s}$ schedule with $3 \mathrm{~s}$ food access. Thus not every positive stimulus presentation yielded reward ensuring later resistance against extinction. Each response to the negative stimuli led to a $0.2 \mathrm{~s}$ darkening of the houselight. While negative stimuli were also presented for a minimum of $30 \mathrm{~s}$, any responses to them led to an obligatory presentation for $10 \mathrm{~s}$ after the response. Thus if the subject persisted in responding, a negative stimulus would stay on indefinitely. Responses to both the positive and the negative stimuli however were counted only during the initial standard $30 \mathrm{~s}$ projection period.

From the fifteenth session onwards further training stimuli (Fig. 1a, right) were introduced at a rate of 4 per session, replacing in each case an equal number of the initial stimuli until the animals had experience with a total of 52 training stimuli. At the same time the darkening of the houselight for incorrect responses was diminished stepwise, so that when the training-only phase ended by session 24 the animals no longer received any feedback upon responding except for the occasional food reinforcements for correct reactions, and for the very rare presentation-extensions for incorrect responses.

Generalization Series: Trials with symmetric and asymmetric patterns that were totally new to the pigeons were now introduced. In each session two such test stimuli were inserted in positions 10 and 20 among a sequence of 28 stimuli randomly chosen from among the repertoire of 52 training stimuli. The responses to the training stimuli had the same consequences as in the preceding training phase. In all but one generalization series one of the two test stimuli was symmetric, the other asymmetric, their order of presentations being randomized between sessions. To control for the effect of the procedure, in one test series (the 'broken' series, Tab. 1), the generalization stimuli shown in alternate sessions were either both symmetric or both asymmetric. Each test stimulus was presented only once for $30 \mathrm{~s}$ to each pigeon. Responses to these stimuli were neither reinforced nor punished but counted separately. Each test series 
consisted of a variable number of consecutive sessions, equal to half the number of the generalization stimuli tested.

Three series, one with each of the subject groups detailed below, involved test stimuli whose geometric style and orientation was similar to that of the training stimuli. One group of animals however viewed them only monocularly (see below). Three other series also employed test patterns in the normal style but they were presented with their figural axes either rotated by $45^{\circ}$ clockwise, or horizontal, or with mixed orientations. Three series used generalization stimuli of non-standard styles, being either of highly irregular, 'Rorschach' shapes, or broken forms, or cross-stich-type patterns of equal surface area. Within another series the test patterns' area varied by as much as a factor of 10 . Finally in yet another series the asymmetric generalization stimuli were repeated patterns, that is shapes constructed by sidewards translation of an element while the symmetric stimuli were constructed by sidewards reflection of the same elements. Examples of the various types of stimuli are shown in Table 1 which also details how many stimuli were used in each series.

Different test series were separately run with one group of 6 pigeons (group A) and another of 4 pigeons (group B). The 4 pigeons of group $C$ trained in the same way as group $A$ and $B$, were equipped with small blocks of metal attached to the skull. To these blocks an eye-cap could be fixed with the aid of a small knurled screw. For surgery the pigeons were anaesthetized with an intramuscular injection of barbiturate/chloralhydrate solution. While the head was held in a stereotactic apparatus, the skin over the skull was slit longitudinally and retracted sidewards. Three anchorage cavities were burred into the skull-bone with a dental drill and the metal block was glued in a standard position with acrylic cement. The skin was drawn together with stitches anteriorly and posteriorly but the block's surface remained exposed. After recovering from the operation for four or five days the animals were retrained for a further 10 sessions wearing over the left eye an opaque hemispherical eye-cup made of gauze and acetate cement. They were then tested according to the same procedure described above.

Acquisition Comparison: The procedure employed was generally similar to that used in the training phase of the preceding experiment. The same apparatus was employed. The subjects were pretrained in a manner analogous to that described earlier. Each session involved the presentation of 20 stimuli in quasi-random sequences according to Gellermann (1933). As before, the standard display time of a stimulus was $30 \mathrm{~s}$ but responses to a negative stimulus could lead to an extension of its presentation. There was no timeout however. Subjects were reinforced with $3 \mathrm{~s}$ food access according to a VI s schedule for responses to the positive stimuli. Separate counters registered the correct and incorrect responses emitted during the standard presentation time. The subjects were run for 25 daily sessions excepting weekends.

Each pigeon had to discriminate two sets consisting of 5 stimuli. All stimuli were drawn from a pool of $20^{\circ}$ patterns, 10 symmetric and 10 asymmetric. 16 pigeons 1

1 Originally 18 pigeons took part, but inadvertently one of them had already participated in the previous experiment. It was subsequently excluded along with its matching partner. 
were divided into two matched groups of 8 . One group of subjects, the 'concept' group had to distinguish sets composed of symmetric stimuli from sets consisting of asymmetric stimuli, that is sets divided along the dimension of interest. The other group of subjects, the control group, discriminated sets of patterns that were not segregated according to this criterion. Two of the control pigeons discriminated symmetric stimuli arbitrarily grouped into two sets, two other control birds dealt with similarly assembled sets of asymmetric stimuli. Four control subjects finally had to distinguish between two arbitrary sets, each of which contained both symmetric and asymmetric patterns. The insets of Fig. 2 illustrate examples of both kinds of stimulus sets, concept and control. Within the groups of pigeons the alternative sets of stimuli were allocated as positive or negative according to a balanced design.

\section{Results}

In the preference tests the subjects slightly favoured the asymmetric patterns. They responded more to the asymmetric than to the symmetric stimuli. The 10 subjects gave a mean $53.7 \%$ of their responses to the asymmetric stimuli with each stimulus presentation yielding an average of 18.6 responses. Eight individuals preferred the asymmetric stimuli and only 2 showed a reverse preference. In 74 of 110 sessions more responses were issued to the asymmetric than to the symmetric stimuli. If the sessions are considered statistically independent of each other this represents a highly significant departure from chance $\left(\chi^{2}\right.$ test, $\left.P<0.01\right)$.

The learning progress in the symmetry/asymmetry discrimination training was assessed by calculating the percent responses to the positive stimuli for each session and subject. Means of these scores for each session were computed separately for the symmetric-positive and asymmetric-positive subject subgroups. Figure 1a shows these means pertaining to group A plotted as a function of the sequence of training sessions. The discrimination learning curves of groups $\mathrm{B}$ and $\mathrm{C}$ were very similar. Learning was rapid and approximately asymptotic performance at above $80 \%$ correct responses was reached by about the tenth training session. The introduction of new training stimuli from session 15 onwards did not lead to any performance decrements. A slight but persistent advantage of the asymmetric positive subgroup over the symmetric positive subgroup was noticeable in all three groups.

The performance on the training stimuli continued at a high level throughout the sessions incorporating the generalization series test trials. Figure $1 \mathrm{~b}$ illustrates this for group A during the first three test series. The left-hand section of Table 1 gives performance averages on the training component for all generalization series and also indicates the corresponding mean number of responses per standard $30 \mathrm{~s}$ stimulus presentation. The generalization performance on the novel test stimuli was similarly assessed by calculating mean percent correct responses to the pair of test stimuli shown in each session. Figure $1 \mathrm{~b}$ shows a plot of this index for the initial three test series of group A. It can be seen that they compare with those on the concurrent training component. A summary of the generalization test results was obtained in two ways. First, the total number of responses to the correct transfer stimuli of the corresponding test series was computed separately for each pigeon and converted into percentages of the total responses to the test stimuli. The means of these 

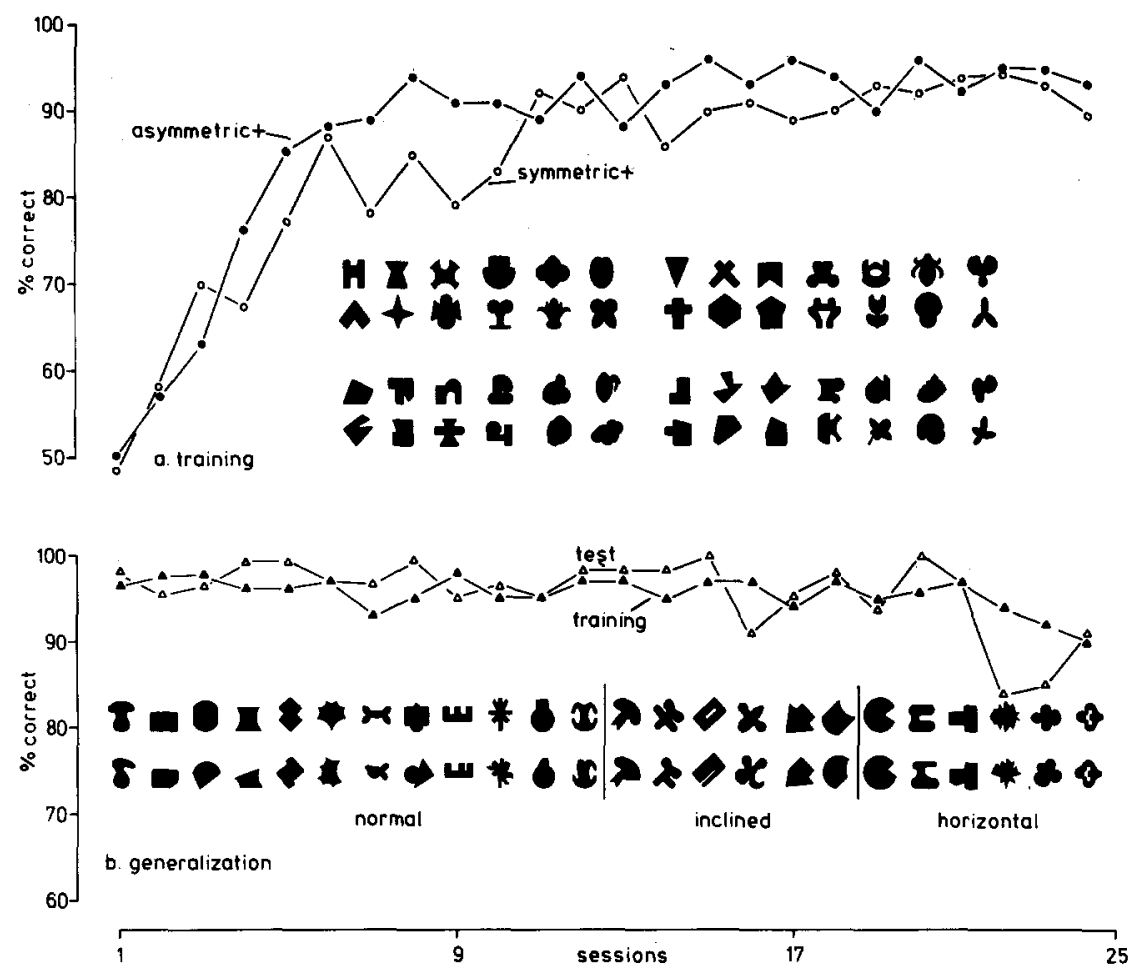

Fig. 1. a Discrimination learning performance for pigeons of group A. Means for subgroups rewarded for responding to symmetric and asymmetric stimuli. Training stimuli, initial set of 24 , left; set of 28 additional stimuli introduced from session 15 onwards, right. b Performance of group A during first three series of sessions incorporating generalization trials. The mean performances with training and novel test stimuli are plotted separately. Stimulus pairs tested in each session are shown

Table 1. Results of sessions incorporating generalization test trials with novel symmetric and asymmetric stimuli. Left side, performance on test stimuli (see text for details). Three groups of pigeons $\mathrm{A}, \mathrm{B}$, and $\mathrm{C}$ took part. The chronological sequence of the test series is indicated by the second digit under the heading 'subject'

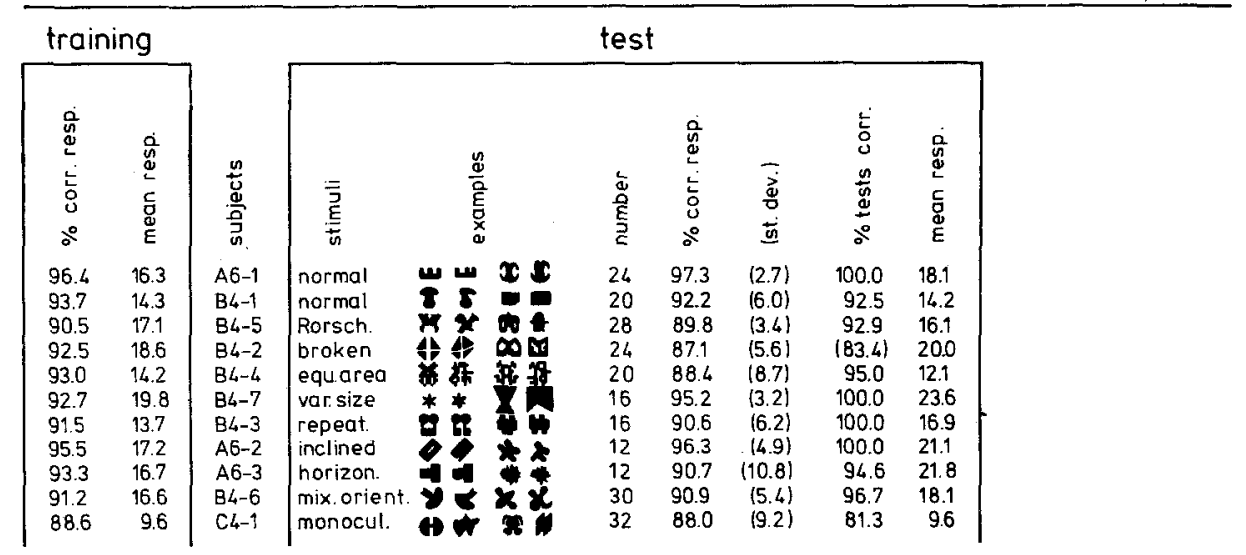


percentages over the subjects and the corresponding standard deviations for the various series are listed in Table 1 . None of the means fell below $85 \%$ correct responses. The standard deviations show that the individuals behaved quite consistently in this respect.

Alternatively, within-session test-trial pairs were scored as correct if the responses to the positive generalization stimulus exceeded those to the negative stimulus. They were scored as incorrect when the reverse was the case, or as it happened on a few occasions when the animals responded equally often to both, or not at all. In the 'broken' series the tests were run according to a modified procedure and this required comparisons of test trial pairs from one session with those of the next. The percentage of test pairs correct are listed in Table 1. Except for two, all the test series yielded more than $90 \%$ correct comparisons, the two exceptions still yielding above $80 \%$ correct. These scores underline the consistency with which the individual stimuli were correctly appraised by the subjects. $\chi^{2}$ tests against chance performance without exception yielded $P<0.01$. The performance with the test stimuli was comparable throughout to the performance with the corresponding training component. The somewhat higher number of mean responses per stimulus overall for the test stimuli as compared with those for the training stimuli is probably explained by the fact that responding to the former was not interrupted by reward as was the case for the latter.

The data recorded in the acquisition comparison experiment were evaluated in terms of percent correct responses produced per session. While in later sessions the animals regularly emitted between 100 and 800 responses, in a few of the early sessions some subjects gave fewer responses. Such sessions were combined until at least 100 responses had accumulated and treated as equivalent to a single session. Mean percent scores were plotted as a function of the progression of sessions separately for the concept and control groups. These learning curves are shown in Fig. 2. It is apparent that the former group showed overall a more efficient acquisition than the latter group. An

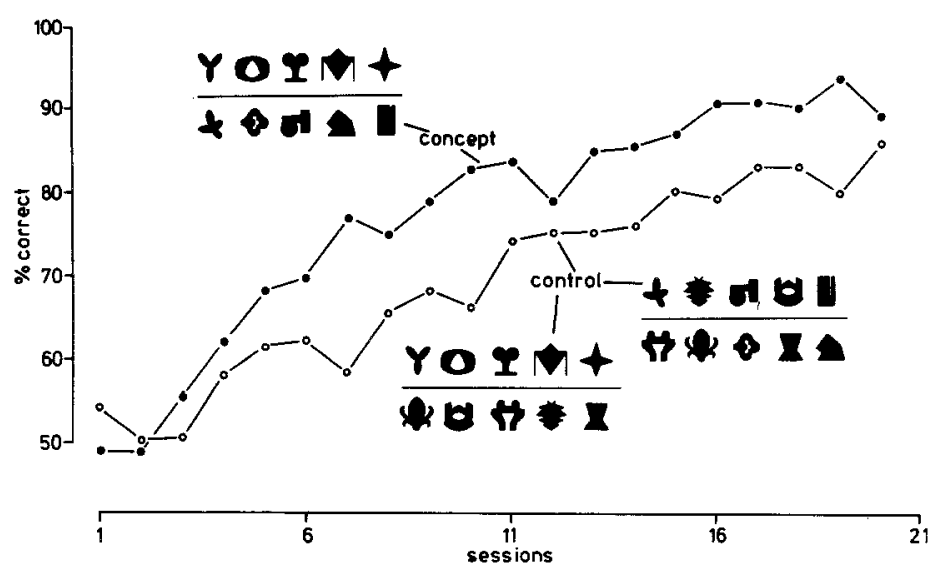

Fig. 2. Mean learning performance of eight pigeons discriminating symmetric and asymmetric sets of stimuli (concept) and eight pigeons discriminating sets of stimuli not classified according to that property (control). Examples of the stimulus set pairs discriminated by the concept and control groups are inserted 
analysis of variance shows that the difference is only just significant $(F$-test, $\mathrm{P}<0.05$ ) due to considerable interindividual variance.

\section{Discussion}

During the discrimination training the pigeons learned to distinguish bilaterally symmetric and asymmetric visual forms quite rapidly. In fact, the acquisition performance compared favourably with that obtained in unpublished experiments where pigeons had to learn to distinguish just two visual patterns under approximately equivalent conditions. This suggests that the animals probably made use of the symmetric-asymmetric stimulus differences as a cue at an early stage of learning. The asymmetric positive subgroups of all three groups of subjects showed a slightly better aquisition. This agrees with similar findings made in our preliminary study (Delius and Habers 1978). The advantage may relate to the weak spontaneous preference by pigeons for asymmetric stimuli upon which we shall comment later.

The fact that the discrimination acquisition proceeded rapidly does not, of course, by itself constitute evidence for the emergence of a symmetry/asymmetry concept. But the generalization tests under extinction conditions demonstrated this in a consistent and convincing manner. Performance on the generalization tests was far better than in the earlier study where there had been evidence that even though generalization was present, the subjects recognized the test stimuli as new: they had responded more hesitantly to the test than to the standard stimuli. This did not seem to be so in the present study: the average number of responses to the test stimuli was equivalent to that to the training stimuli. The larger number of training stimuli (52 as compared with 30 ) and the modified procedure used in this study may well have made a recognition of new stimuli more difficult.

The stimuli of the initial generalization series, 'normal', were purposely similar in geometric style to the standard training stimuli. The transfer to them was very high. The stimuli used in other test series differed more markedly in design. The stimuli of the 'Rorschach' series for example were quite distinct from the training stimuli in being of a highly irregular, haphazard outline. A good generalization performance was nonetheless maintained. The 'broken' series of test stimuli consisted of patterns built up from several juxtaposed elements, each of these often being asymmetrical in itself but adding to an overall symmetrical pattern in the relevant stimuli. It is obvious that the animals were not much disconcerted by this inconsistency and recognized the symmetry of the whole patterns. Similarly, the aberrant testing paradigm that was used in this instance did not disrupt the generalization performance.

At this point we can already conclude that pigeons are capable of recognizing visual symmetry from non-symmetry in a generalizing way after they have been trained to discriminate on sets of examplars. This conclusion coincides with that reached in our preliminary study (Delius and Habers 1978). Insofar as symmetry and asymmetry can be said to be abstract properties of visual forms the pigeons may be supposed to have applied a concept-like criterion to execute this classification. Symmetry can thus be added to the growing list of perceptual concepts which pigeons are able to master. Among such concepts one may list pigeon, tree, oakleaf, water, 
fish, people, person, man-made landscape, letter A, and others (Herrnstein and Loveland 1964, Siegel and Honig 1970, Poole and Lander 1971, Lubow 1974, Morgan et al. 1976, Herrnstein et al. 1976, Herrnstein and de Villiers 1980).

It must now be asked what kind of perceptual mechanism is responsible for this performance. The unlikely possibility that the animals might use as a cue a tendency for symmetrical figures to have a larger surface area than comparable asymmetrical ones was considered. The 'equal area' series was designed to control for this while at the same time testing stimuli of yet another style. The generalization performance remained excellent. The size of the patterns was then deliberately varied in the 'varied sizes' test series, the larger test patterns being about twice, the smaller about half the size of the usual patterns. The generalization performance remained unaffected.

The 'repeated' series was inserted to counter the hypothesis that symmetric patterns are recognized on the basis of the simple redundancy that is inherent to them (Attneave 1955). Asymmetric forms that consist of an element replicated after sidewards translation are at least as redundant as the equivalent symmetric forms and should thus be confused with the latter. Pigeons like humans (Corballis and Roldan 1974), have no difficulty in distinguishing these two kinds of stimuli, so that a recognition according to a primitive redundancy hypothesis is not tenable. This does not imply that redundancy might not be important in mediating the aesthetic and salience effects that symmetric shapes seem to have for humans (Weyl 1952, Howe 1980).

It is possible that the recognition of symmetry may be connected with the bilateral symmetry of the visual structures of pigeons and of course many other organisms. A model of how the anatomical symmetry could mediate the recognition of the symmetry of visual patterns by pigeons is presented in Fig. 3. It derives from one proposed in connection with an unorthodox interocular transfer shown by these birds. A pigeon that learned to respond to a particular pattern seen through one eye, when tested through the other tends to choose the mirror image of the original pattern rather than the pattern itself (Mello 1965, but see Watanabe 1979). This can be explained by assuming a homotopic (rather than veridical) transfer of information about shapes across the interhemispheric (or in the pigeon possibly intertectal) commissures (but see Cuénod 1974, Voneida and Mello 1975). In the present
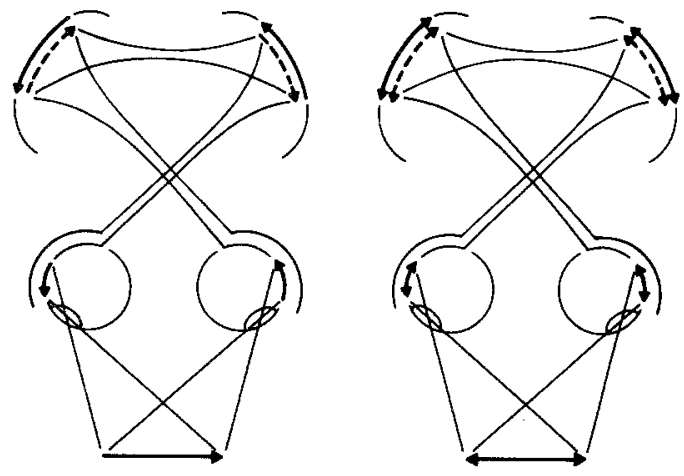

Fig. 3. Hypothetical recognition by pigeons of an asymmetric stimulus (arrow) and symmetric stimulus (double arrow) with the aid of a homotopic intertectal transfer. Direct and transferred tectal stimulus representations coincide in the case of a vertically symmetric stimulus, but not in the case of an asymmetric stimulus 
context such an arrangement yields matching of both the direct and transferred neural representations only of symmetric visual patterns. Such matching could be the basis of symmetry recognition. An analogous, somewhat more complex (because of the hemidecussation of the optic nerves present in mammals) model of symmetry recognition can be imagined to apply to humans (Corballis and Beale 1976, Achim and Corballis 1976). Notice, however, that such models only work with bilaterally symmetric patterns whose axis coincides with the symmetry plane of the organism's head. For humans there is indeed evidence that symmetry recognition is better when this condition is met (Julesz 1971, Palmer and Hemenway 1978, Barlow and Reeves 1979).

The results of the series 'inclined', 'horizontal', and 'mixed' orientation suggests that this may not be true for pigeons. The possibility that pigeons might have compensated the differing axis orientations of the symmetric stimuli with head movements was excluded by observing them in the mixed orientation series. While the head position shortly before responding to the stimuli could vary by 30 degrees either way from the vertical, this did not relate to the symmetry axis orientation of the stimuli in question. This insensitivity to symmetry axis orientation may be related to the unusual ability that pigeons appear to have for recognizing visual patterns regardless of their orientation, which has been discussed elsewhere (rotational invariance, Hollard and Delius 1982).

The stongest test of the hypothesis that symmetry recognition is not founded on the gross anatomical symmetry of the visual system of the pigeons is the 'monocular' series. In the context of the above model and due to the virtually total crossing of the optic nerve in birds (Cowan et al. 1961, Réperant 1973) it is obvious that occlusion of one eye should have drastic consequences for the recognition of symmetry. The result of the test series shows that this is not so: the animals continue to discriminate correctly. The possibly slightly worse performance is almost certainly due to the lower response rates shown both towards the training and test stimuli. The reluctance of pigeons to peck under monocular conditions is probably due to a consequential lack of binocular depth perception that very likely hinders an adequate control of peck-depth.

None of the classical theories of symmetry recognition seems to explain the pigeon's and, it would seem, also human performance in symmetry recognition. An alternative mechanism is called for. The mammalian (DeValois and De Valois 1980), and also the avian (Jassik-Gerschenfeld and Hardy 1981) visual systems perform a local Fourier analysis at an early stage of their information processing. Information about the phase relationships of the spatial frequency components of visual scenes is essential for pattern perception. It is instructive to examine bilateral symmetry from this point of view (Fig. 1; see also Julesz and Chang 1979). At the axis of a symmetric patterns the phase relationships of all the component spatial frequencies are either $0^{\circ}$ (in-phase) or $180^{\circ}$ (counter-phase). A non-symmetrical pattern does not yield these relationships anywhere. Translated in terms of neuronal mechanisms, lightbar and dark-bar detectors for various bar widths that coincide in terms of the position of their receptive field axes should facilitate each other in some way, whereas they should inhibit similar bar detector ensembles with neighbouring receptive fields. Some indirect evidence that this may be so is available for humans (Atkinson and 

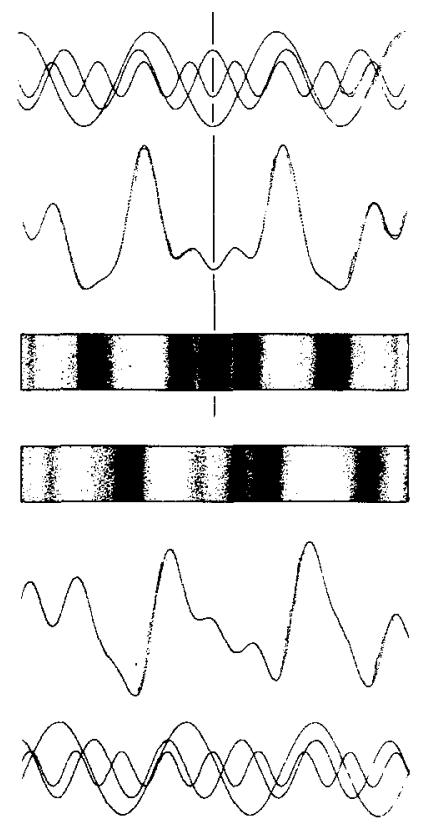

Fig. 4. Symmetry of visual patterns in terms of spatial Fourier analysis. A symmetric and an asymmetric frieze is represented as a brightness profile that is then decomposed into sine waves. At the symmetry axis the components are all in-phase $\left(0^{\circ}\right)$ or antiphase $\left(180^{\circ}\right)$. Such a phase relationship is not present anywhere in an asymmetric pattern

Campbell 1974). Alternatively, bar- and edge-detector neurons that have coinciding receptive fields may reciprocally inhibit each other and thus function as symmetry/ asymmetry detectors. Again, this seems possible in mammals (Pollen and Ronner 1981).

The question is then how such neuronal symmetry filters come to develop during ontogeny. We doubt that we taught our pigeons a symmetry concept but rather believe that they only learned to apply an already available competence. The results of the preference tests suggest that pigeons, without having been specifically trained, can discriminate symmetric from asymmetric patterns. The preference shown by the pigeons is incidentally the reverse of that evinced by humans who tend to strongly favour symmetric stimuli (Eisenman and Gellens 1968, Szilagyi and Baird 1977, see also Rensch 1958, jackdaws, Corvus monedula). The results of the acquisition comparison experiment also support the pre-existence of a discriminative capability. The presence of a symmetry/asymmetry difference between two small sets of stimuli aided discriminative learning from a very early stage onward seemingly precluding that a generalized symmetry/asymmetry recognition could have arisen through the specific experience provided by the experiment. We suspect that the mechanisms responsible for the distinction in question develop very early in ontogeny and largely independently of specific and differential experience with symmetric and asymmetric patterns. The same seems to be true for humans (Bornstein et al. 1981). At the very least we would estimate that whatever learning that might be necessary is of a very 'prepared' kind (Seligman 1970). This implies that we may be dealing with an innate or nearly innate symmetry/asymmetry concept, an extremely natural category in the terms of Rosch (1973). It must be decided whether it is semantically convenient to 
encompass this kind of super-generalization within the term concept. We would like to criticize here, incidentally, the rather indiscriminate usage that Herrnstein and associates make of the term 'natural'. It seems unwarranted, for example, to characterize as natural the category 'fish' learned by pigeons (Herrnstein and de Villiers 1981) in view of the fact that pigeons as a thoroughly non-aquatic species are unlikely to ever have had, either during their more recent phylogeny or ontogeny any more than fleeting experience with fish.

The assumption that symmetry recognition is due to largely innate neural filters raises the issue of what kind of selection pressures might have brought about the evolution of symmetry filters. Curio (1976) considered the possibility that symmetry detection might aid predators in finding comouflaged prey (see Menne and Curio 1978) and we have considered whether this could also extend to pigeons searching for grains (Delius and Habers 1978). For humans it has been said that the all-important recognition of faces is aided by symmetry salience (see also Barlow and Reeves 1979 for rather original alternative views on this point). Nonetheless, we choose to doubt that symmetry recognition mechanisms per se have ever been primarily selected for in any animal group. Rather we suspect that symmetry filters have emerged as a by-product of selection for more general functions. A neural system that has to deal homogeneously with surface information (the retinal image) can hardly have anything other than a multiply symmetrical structure. On the other hand the detection of direction of visual movement obligatorily requires asymmetrical structures. The requisites for a symmetry/asymmetry discrimination may thus have been selected for the fulfilment of very basic but nonetheless extraneous system capacities. This brings the perceptual singularity of visual symmetry into the neighbourhood of many visual illusions. They also often appear to be 'unintended' side-effects of mechanisms that subserve quite useful, real tasks.

It is nevertheless remarkable that visual systems that are as phylogenetically divergent as that of the pigeon (midbrain-based) and that of man (endbrain-based) should apparently have evolved mechanisms with similar symmetry recognition performances.

Acknowledgements. The research was supported by the Deutsche Forschungsgemeinschaft through its Sonderforschungsbereich 114 and by the Research Fund of the Ruhr-Universität. The special cooperation of Detlef Wolf, Christoph Werner and Claudia Thöne is particularly acknowledged. We also thank D. Hagenkötter, A. Lohmann, and J. Emmerton for essential assistance in various matters.

\section{References}

Achim A, Corballis MC (1976) Mirror-image equivalence and the anterior commissure. Neuropsychol 15:475-478

Atkinson J, Campbell FW (1974) The effect of phase on the perception of compound gratings. Vision Res 14:159-162

Attneave F (1955) Symmetry, information and memory for patterns. Am J Psychol $68: 209-222$

Barlow HB, Reeves BC (1979) The versatility and absolute efficiency of detecting mirror symmetry in random dot displays. Vision Res 19:783-793 
Bornstein MH, Ferdinandsen K, Gross CG (1981) Perception of symmetry in infancy. Develop Psychol 17:82-86

Corballis MC, Beale IL (1976) The psychology of left and right. Lawrence Erlbaum Associates, Hillsdale, NJ

Corballis MC, Roldan CE (1974) On the perception of symmetrical and repeated patterns. Percept Psychophys 16:136-142

Cowan WM, Adamson L, Powell TPS (1961) An experimental study of the avian visual system. J Anat 95:545-563

Cuénod M (1974) Commissural pathways in interhemispheric transfer of visual information in the pigeon. In: Schmitt FO, Worden FG (eds) The neurosciences, third study program. MIT Press, Cambridge, Ma

Curio E (1976) The ethology of predation. Springer, Berlin Heidelberg New York

Delius JD, Habers G (1978) Symmetry: Can pigeons conceptualize it? Behav Biol $22: 336-342$

DeValois R, De Valois KK (1980) Spatial vision. Ann Rev Psychol 31:309-341

Eisenman R, Gellens HK (1968) Preference for complexity-simplicity and symmetryasymmetry. Percept Mot Skills 26:888-890

Gellermann LW (1933) Chance orders of alternating stimuli in visual discrimination experiments. J Genet Psychol 42:206-208

Herrnstein RJ, Loveland DH (1964) Complex visual concept in the pigeon. Science $146: 549-551$

Herrnstein RJ, Loveland DH, Cable C (1976) Natural concept in the pigeon. J Exp Psychol Anim Behav Process 2:258-302

Herrnstein RJ, DeVilliers PA (1980) Fish as a natural category for people and pigeons. Psychol Learn Motiv 14:59-95

Hollard VD, Delius JD (1982) Rotational invariance in visual pattern recognition by pigeons and humans. Science (in press)

Howe ES (1980) Effects of partial symmetry, exposure time and backward masking on judged goodness and reproduction of visual patterns. Quart J Exp Psychol $32: 27-55$

Jassik-Gerschenfeld D, Hardy O (1981) Single-cell response to bar-width and to sinewave grating frequency in the pigeon optic tectum. Vision Res 21:745-747

Julesz B (1971) Foundations of cyclopean perception. University of Chicago Press Chicago

Julesz B, Chang JJ (1979) Symmetry perception and spatial-frequency channels. Perception 8:711-718

Lubow RE (1974) High-order concept formation in the pigeon. J Exp Anal Behav $21: 475-483$

Mello NK (1965) Interhemispheric reversal of mirror-image oblique lines following monocular training in pigeons. Science 148:252-254

Menne M, Curio E (1978) Untersuchungen zum Symmetriekonzept bei Kohlmeisen (Parus major L). Z Tierpsychol 47:299-322

Morgan MJ, Fitch MD, Holman JG, Lea SEG (1976) Pigeons learn the concept of an 'A'. Perception 5:57-66

Palmer SE, Hemenway K (1978) Orientation and symmetry. Effects of multiple, rotational and near symmetries. J Exp Psychol Human Percept Perform 4:691-702 
Pollen DA, Ronner SF (1981) Phase relationships between adjacent simple cells in the visual cortex. Science 212:1409-1411

Poole J, Lander DG (1971) The pigeon's concept of a pigeon. Psychon Sci 25:157158

Rensch B (1958) Die Wirksamkeit ästhetischer Faktoren bei Wirbeltieren. Z Tierpsychol $15: 447-461$

Réperant J (1973) Nouvelles données sur les projections visuelles chez le pigeon (Columba livia). J Hirnforsch 14:151-187

Rosch EH (1973) Natural categories. Cognit Psychol 4:328-350

Seligman MEP (1970) On the generality of the laws of learning. Psychol Rev 77: 406-418

Siegel RK, Honig WK (1970) Pigeon concept formation: Successive and simultanous acquisition. J Exp Anal Behav 13:385-390

Szilagyi PG, Baird JC (1977) A quantitative approach to the study of visual symmetry. Percept Psychophys 22:287-292

Voneida TJ, Mello NK (1975) Interhemispheric projections in pigeon. Brain Behav Evol 11:91-108

Watanabe S (1979) Mirror image discrimination with each eye in pigeons. Physiol Behav 22:331-337

Weyl H (1952) Symmetry. Princeton University Press, Princeton 